training opportunities in its management are limited. Trainees should be aware of the different methods and their limitations.

\section{Compliance with ethical standards}

Conflict of interest The authors declare that they have no conflict of interest.

\section{References}

1. Davison JA. Capsule contraction syndrome. J Cataract Refract Surg. 1993;19:582-9.
2. Tsinopoulos I, Symeonidis C, Frangou E, Dimitrakos SA. Capsule contraction syndrome in eight cases of hydrophobic one-piece intraocular lens implantation. Clin Exp Optom. 2008;91:469-72.

3. Yeh PC, Goins KM, Lai WW. Managing anterior capsule contraction by mechanical widening with vitrector-cut capsulotomy. J Cataract Refract Surg. 2002;28:217-20.

4. Panagopoulos A, Chalioulias K, Kirkby GR. A new approach in the surgical management of anterior capsular phimosis syndrome. Ophthalmic Res. 2009;42:221-3.

5. Wilson RP, Gupta R. Focal corneal decompensation caused by an anterior capsulotomy remnant. J Cataract Refract Surg. 1997;23: $1273-4$.

\title{
Comment on: Assessment of patient-reported outcome and quality of life improvement following surgery for epiphora
}

\author{
Valerie Juniat $^{1} \cdot$ Sarju Athwal ${ }^{1} \cdot$ Adeela Malik $^{2} \cdot$ Mona Khandwala $^{1}$ \\ Received: 11 January 2018 / Accepted: 12 February 2018 / Published online: 18 May 2018 \\ (c) The Royal College of Ophthalmologists 2018
}

\section{Dear Editor,}

We read with great interest the article by Sipkova et al. [1] entitled "Assessment of patient-reported outcome and quality of life improvement following surgery for epiphora" in the December 2017 issue of Eye. We have reviewed the patient-reported outcomes of our own patients who have undergone surgery for epiphora. We would like to share our experience with readers.

Sipkova et al. [1] discussed the relevant limitations of the Glasgow Benefit Inventory (GBI), Munk symptoms score [2], and Lac-Q questionnaire. In our study, we prospectively enrolled 17 patients (20 eyes) who underwent lateral tarsal strip procedures for epiphora and lower lid laxity between June 2014 and September 2015. All 17 patients (20 eyes) completed the Munk score preoperatively and at 3 months

Valerie Juniat

vjuniat@doctors.org.uk

1 Maidstone and Tunbridge Wells NHS Trust, Hermitage Lane, Maidstone, Kent ME16 9QQ, UK

2 Moorfields Eye Hospital NHS Trust, 162 City Road, London EC1V 2PD, UK postoperatively. Patients subjectively scored their epiphora severity based on the $0-4$ scale, where. is no watering and. is constant watering. Statistical analysis was carried out using paired $T$-test. Average Munk score prior to lid surgery was $4.65 \pm 0.49 \mathrm{SD} \quad($ median $=5)$ and $2.05 \pm 1.05 \mathrm{SD}$ (median $=2$ ) postoperatively. The mean difference in preoperative and postoperative Munk score was $2.6 \pm 1.05 \mathrm{SD}$ (median $=3, p<0.001,95 \%$ CI 2.11-3.09). Only one patient had no change in their Munk score following lateral tarsal strip surgery, scoring. both pre and postoperatively.

Our study findings compare with those from Sipkova et al. [1], where their patients also showed significant improvement in Munk score following lid-tightening surgery $(p<0.001)$. In our experience, the Munk score can be applied realistically during busy outpatient clinical settings in view of its ease of completion. However, the Munk score has its limitations in assessing the impact of epiphora on specific daily tasks. For example, our single patient who did not show improvement in Munk score may have had very severe frequency of wiping from 50 times. day preoperatively (Munk score of 4) to 20 times. day postoperatively (Munk score of 4), an improvement for the patient. We therefore support Sipkova et al. [1] in their 
notion for the development of. validated PROM questionnaire for use following non-DCR, surgical intervention for epiphora.

\section{Compliance with ethical standards}

Conflict of interest The authors declare that they have no conflict of interest.

\section{References}

1. Sipkova Z, Vonica O, Olurin O, Obi EE, Pearson AR. Assessment of patient-reported outcome and quality of life improvement following surgery for epiphora. Eye. 2017;31:1664-71.

2. Munk PL, Lin DT, Morris DC. Epiphora: treatment by means of dacryocystoplasty with balloon dilatation of the nasolacrimal drainage apparatus. Radiology. 1990;177:687-90.

\title{
Exploring correlations between change in visual acuity following routine cataract surgery and improvement in quality of life assessed with the Glasgow Benefit Inventory
}

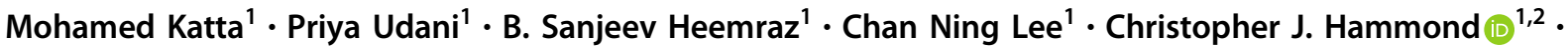 \\ Omar A. Mahroo $1,2,3,4$
}

Received: 15 January 2018 / Accepted: 25 April 2018 / Published online: 18 May 2018

(c) The Royal College of Ophthalmologists 2018

The Glasgow Benefit Inventory (GBI) [1] assesses patientperceived changes in quality of life following an intervention by means of 18 questions; the score ranges between a minimum of -100 (maximal detriment) to +100 (maximal benefit). As it is not procedure-specific, it can potentially be used to compare changes in quality of life following different interventions. Originally developed for use in otolaryngology, it has also been used in a range of oculoplastic procedures [2,3], and, more recently, we have deployed it after routine cataract surgery [4]. In the latter study, the questionnaire was administered to 109 patients, and significant improvement in quality of life was demonstrated with a positive GBI score.

Omar A. Mahroo

omar.mahroo@kcl.ac.uk

1 Department of Ophthalmology, St Thomas' Hospital, London SE1 7EH, UK

2 Department of Ophthalmology, King's College London, St Thomas' Hospital Campus, Westminster Bridge Rd, London SE1 7EH, UK

3 UCL Institute of Ophthalmology, University College London, 1143 Bath St, London EC1V 9EL, UK

4 Retinal Service, Moorfields Eye Hospital, 162 City Rd, London EC1V 2PD, UK
In the present study, we retrospectively retrieved visual acuity data for these patients (from the patient record) to explore correlation between change in visual acuity (decimalised) and GBI score. Patients for whom the preoperative or post-operative visual acuity was not available were excluded. 91 patients (83.5\%) were included: 48 were female; 53 were Caucasian, 30 Black, 3 Asian (5 had unrecorded ethnicity). The mean (SD) age was 71 (11) years.

Table 1 shows the main findings, and Fig. 1 plots GBI scores against change in visual acuity. For the whole cohort GBI score correlated positively with visual acuity with a Pearson correlation coefficient of $0.35\left(p=7 \times 10^{-4}\right)$. For first eyes, correlation was stronger than for second eyes. The visual acuity improvement for first eyes was greater than that for second eyes as might be expected (given the usual practice of operating on a worse seeing eye first). Importantly, an improvement in quality of life (positive GBI score) was seen after both first and second eye surgery, with no significant difference in scores, despite the difference in change in visual acuity. Mean pre and post-operative visual acuity did not differ significantly by ethnicity.

Our findings quantify the correlation between quality-of-life improvement, as measured by the GBI, and change in visual acuity. A significant correlation was seen. However, the strength of the correlation was 